\title{
CLOUD COMPUTING AND INTERNET OF THINGS CONCEPTS APPLIED ON BUILDINGS DATA ANALYSIS
}

Florin-Adrian HEBEAN - PhD Student, Technical University of Civil Engineering Bucharest, Faculty of Building Services, e-mail: hebeanflorin@gmail.com

Sorin CALUIANU - Professor - PhD Director, Technical University of Civil Engineering Bucharest, Faculty of Building Services, e-mail: s_caluianu@yahoo.com

\begin{abstract}
Used and developed initially for the IT industry, the Cloud computing and Internet of Things concepts are found at this moment in a lot of sectors of activity, building industry being one of them. These are defined like a global computing, monitoring and analyze network, which is composed of hardware and software resources, with the feature of allocating and dynamically relocating the shared resources, in accordance with user requirements. Data analysis and process optimization techniques based on these new concepts are used increasingly more in the buildings industry area, especially for an optimal operations of the buildings installations and also for increasing occupants comfort. The multitude of building data taken from HVAC sensor, from automation and control systems and from the other systems connected to the network are optimally managed by these new analysis techniques. Through analysis techniques can be identified and manage the issues the arise in operation of building installations like critical alarms, nonfunctional equipment, issues regarding the occupants comfort, for example the upper and lower temperature deviation to the set point and other issues related to equipment maintenance. In this study, a new approach regarding building control is presented and also a generalized methodology for applying data analysis to building services data is described. This methodology is then demonstrated using two case studies.
\end{abstract}

Keywords: cloud computing, data analyses, bms, building automation, internet of things

\section{Introduction}

Along with the development of new technologies and the development of communications networks, new concepts like Cloud Computing and Internet of Things have been emerged. Appeared and debated for the first time by the British researcher Kevin Ashton in 1999 [1], the Internet of Things concept is defined to be a physical components network (things), like electronic equipment, software applications, sensors and internet connectivity with the possibility of communication between them, without the intervention of a human operator. Acquired data from the network can be collected, stored and analyzed.

In present, the Internet of Things and Cloud Computing concepts are applied in many areas of activity, the main ones being the automotive industry, smart cities, health sectors, oil and gas industry and the field of buildings and residential dwellings [2].

In the residential dwellings area, the Cloud Computing and Internet of Things concepts, bring the increase of life quality through the connection and intercommunication of various household appliances, building automation and security systems, lighting systems and other electronic equipment.

The electronic infrastructure (brain) of the building that drives and monitors the operation of the associated equipment and facilities is known as BMS - Building Management System. The BMS concept of a building encompasses all appliances, equipment, local automation systems (hydraulic, heating, ventilation and air-conditioning, elevation, lighting, fire prevention and extinction, access control, surveillance, anti-burglary, etc.) and communication networks that supervise and control the operation of the building's facilities. Building Management Systems - 
BMS implements efficient energy use, programs under fire safety, security and reduces maintenance costs. BMS is a modern automation system with a hierarchical architecture and distributed on two or three levels. The main elements are the central computer (PC Workstation) and the controllers required to the various type of equipment and installation. The transmission of information between them and the computer and vice versa is done in real time through a communication network. The controllers are electronic devices equipped with a microprocessor and have implemented modern operating algorithms. The communication network also provides the flow of information between controllers and during the temporary failure of the central computer, they work together for the building operation.

The main function of the BMS systems is to control the HVAC installation. The HVAC installation is the abbreviation for Heating, Ventilation and Air Conditioning systems. The main parameters which are controlled and maintained in the HVAC systems are temperature, humidity, air flow and air filtering. Managing these parameters, HVAC systems have the purpose of providing thermal comfort and air quality in standard parameters for the occupants of the building. Heating - the $\mathrm{H}$ in HVAC is the process of generating and producing warmth for the building. Ventilation - the V in HVAC is the process of discharge and exhaust air for building spaces, to maintain the air quality and the level of $\mathrm{CO} 2$ and also to control the temperature. Air Conditioning - the AC from HVAC is the process of removing heat from the interior of an occupied space in order to improve the comfort of occupants.

Related to offices and commercial buildings, the BMS systems that connect HVAC installations, lighting systems, shading systems and low current systems become more performing. The occupants comfort and the energetic performance of the building are considerable increased with the Cloud Computing and Internet of Things implementation.

The fault detection process analyzes the measured signals such as vibration, noise, acoustic sound, pressure or bases on the analytical parameters in order to generate the faulty symptoms. The faulty symptoms are the input of the fault diagnosis process that determines the size, type and location of the system fault. These can be analytical or heuristic. [3].

Like in the industrial sectors of activity, in the building field, there have been created some data analytics platforms, one of the most used of these being SkySpark developed by SkyFoundry [4].

The platform has been developed as a software architecture solution, end to end type. The scope of this data analysis platform is to give a correct and efficient perspective about the building comportment, by aggregation, extraction, and analysis of data provided by sensors, equipment and products. The management, search, analysis and prediction of data are achieved by accessing one unified software platform. Also, the software platform allows the building automation systems, the energy and water meters, the equipment and sensors to communicate data about their function and also about their operating environment. Accessing the building operation data at any time and from any place, makes possible the augmentation of the building energetic performance, occupancy comfort and reduces the maintenance costs. Combining a vast library of standard analytical features and enabling users to develop their own functions, the software platform gives the possibility to the experts to develop their own functions and analytics rules which will be applied afterwards on data provided by equipment and sensors [5].

The ultimate goal of the analytics software platform is to retrieve data from any kind of system and any kind of equipment, regardless the manufacturer. In this way it was developed the Haystack standardization [6], which offers the possibility to tag the data so as to be recognized by the software analytic platform and also to apply rules and function for this data.

The first objective of this paper is to explain how data is extracted from the standard building management and automation system and pushed to the software analytics platform, using 
Haystack concepts. For this purpose, the paper is presents the virtualization and tagging process and also, a diagram of this process.

The second objective of the paper is to create and develop new analytics function applied on HVAC systems. To achieve this, the standard libraries of the SkySpark analytics platform and also the programing language behind the analytics platform called Axon will be used. A set of analytics rules regarding occupants comfort, equipment proper operation and building energetic performances are then created.

The last objective of the paper is to apply the developed rules to a set of data, provided by a specific building. The rules will be applied for one week data series and the results are explained and analyzed.

\section{Data virtualization for Cloud Computing}

To enable the development and enforcement of the analytics rules for a large number of and for any building type, the data provided to the analysis platform for each location is aligned and mapped in accordance with the specific input requirements for each analytics rules set. This process is accomplished using two levels of abstraction. The first abstraction level involves the use of the industry standard for data tagging called Project Haystack [6], to describe the data that arrives in the software analysis platform. The Haystack standard provides a predefined ontology for describing the building installations data. An ontology defines how elements are arranged in a hierarchy or structure and how these elements interact and relate to one another [7]. Elements have defined attribute values or sets of attributes. In practical situations, using the concepts of Haystack terminology, the input data are tagged. The tags can be read by the computer and also by the human and allow the rules to be applied to the data. If permutations of elements occur in time, both new labels and new relationships will be added.

The second level of abstraction is the link between tagged data and rules and serves to provide the input data of the underlying functions. This is done with help desk libraries. Help functions have the role of selecting the best available data from the input data set and also making the rules work even with a minimum data set. An example of this kind of function is presented in Fig.1. The help function in the example below provides an outside relative humidity history and provides this value to an analytics rule.

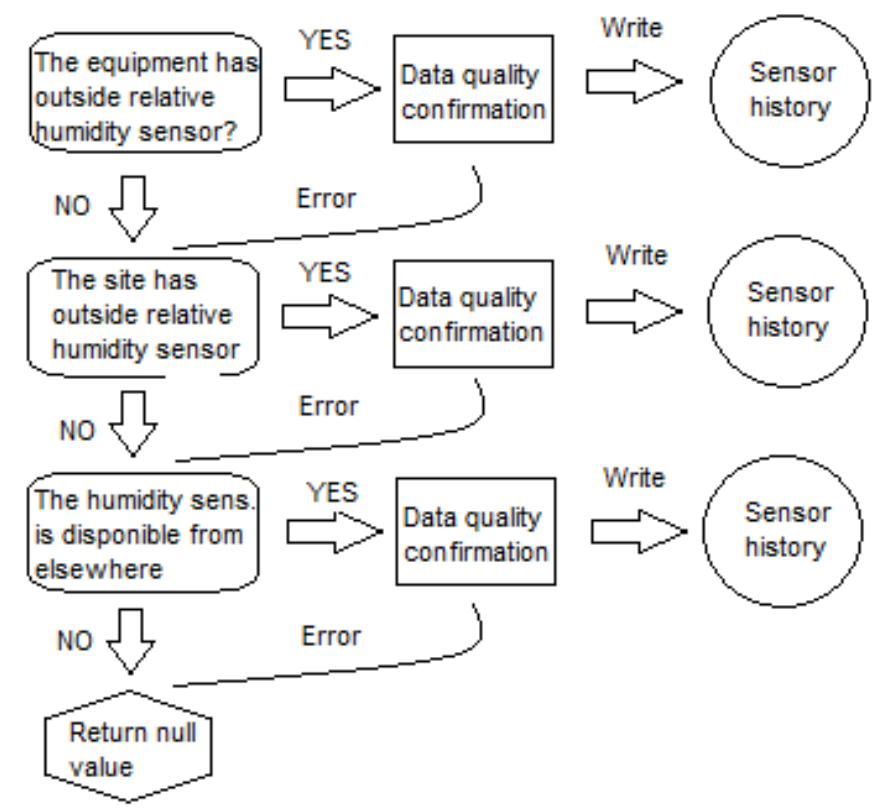

Fig.1 - Internal logic of help function for outside relative humidity history 


\subsection{Haystack virtualization process model}

The Internet of Things (IoT) and Cloud Computing concepts are defined as a network of networks, being an interoperable system for different physical and soft networks. Working with a diversity and a huge amount of information, data acquired in the cloud must be virtualized and tagged. For virtualization of BMS and other building systems data, an ontology based on the Haystack concept is used, divided into three modules: the meta model, the vocabulary and the domain model.

The meta model aims to assign a label to the vocabulary of each input or equipment, so that the input data and the equipment to get a general character and can be easily identified in the analysis platform, to be integrated into various functions and rules. The vocabulary includes a diverse set of labels that will be assigned to input data and also to the equipment. The domain model is the entire assembly that is controlled by the systems, including measured values, setpoints, and equipment. The model of the virtualization and tagging process is presented in Fig.2.

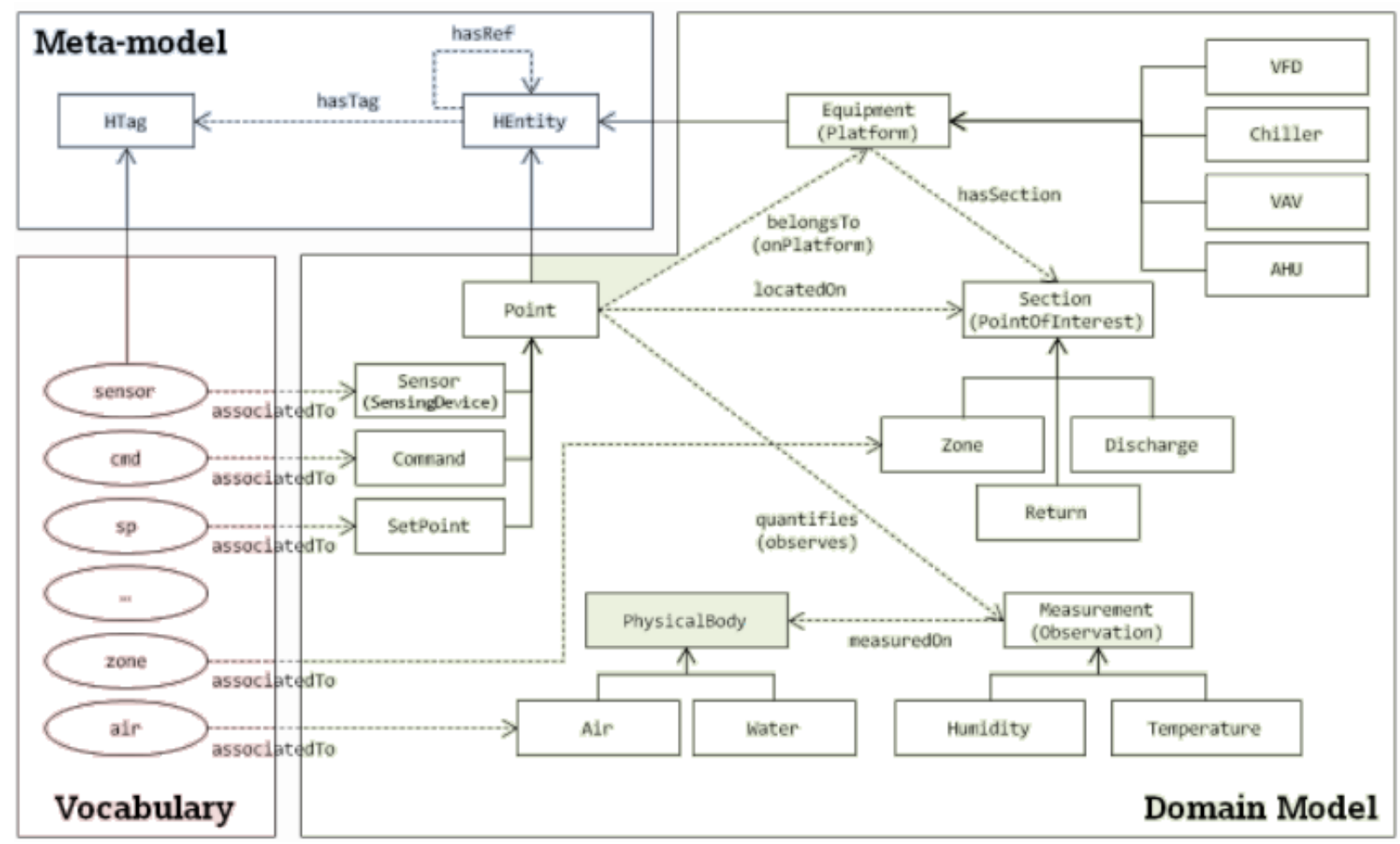

Fig.2 - Haystack virtualization process model [8]

\section{Definition of software analytics rules in the Cloud Computing platform}

Almost all building zone controllers need to adjust and manage three important parameters that affect the occupants comfort: temperature, relative humidity and air quality. To maintain these three parameters into the users or building managers predefined limited, the automation controllers need to drive the heating and cooling processes, the humidification and dehumidification and fresh air supply and vicious air exhaust processes. In almost all cases, the comfort parameters mentioned above are not maintained correctly and the occupant's comfort is affected.

Using the Cloud Computing software analytic platforms, the building managers have, at any time, an overview on each comfort parameter and any deviation from setpoint is detected and reported. Also, using software analytic platforms, all the parameters and equipment commands are added into a history and the historical data is available anytime and everywhere. In this paper there are defined analytic rules, namely inefficient heating and inefficient cooling, regarding occupants of the building thermal comfort. These rules are following any deviation from the 
predefined setpoint. The other main objective at this moment in building industry is to increase the energetic performances. For this purpose a rule which detect the abnormal energy consumption will be presented.

\subsection{Defined analytic rules related to occupants thermal comfort}

In terms of maintaining the temperature comfort parameter, three analytical rules are defined, namely: zone temperature setpoint untouched, heating inefficiency and cooling inefficiency. The set of rules will have input temperature measured by the zone sensor and the users defined setpoint as well as the controller's output values, values that act the heating and cooling actuators.

\subsubsection{The zone temperature setpoint untouched analytic rule}

For stationary regime, this analytics rule check how close is the actual temperature measured by the zone temperature sensor to the user defined setpoint. If this difference is bigger than a predefined value, the analytical rule result is an operating system error. In composing this rule, it is defined a threshold of error, for the difference between the setpoint and the measured temperature. Excepting the transitory regime, the rule will be operational when the cooling or heating equipment will work and is a requirement for heating or cooling in the zone.

The equation underlying this rule is the following:

$$
\begin{aligned}
& \text { Setpoint-err } \leq \text { Temperature } \leq \text { Setpoint }+ \text { err; } \\
& \text { err }=\frac{\text { Offset }}{2} \sim 1^{\circ} \mathrm{C} \text {; }
\end{aligned}
$$

where:

Setpoint-the temperature request by user;

Temperature-the temperature measured by building temperature sensor;

Offset-the accepted deviation from Setpoint;

The diagram of the zone temperature setpoint untouched analytic rule process is presented in Fig.3.
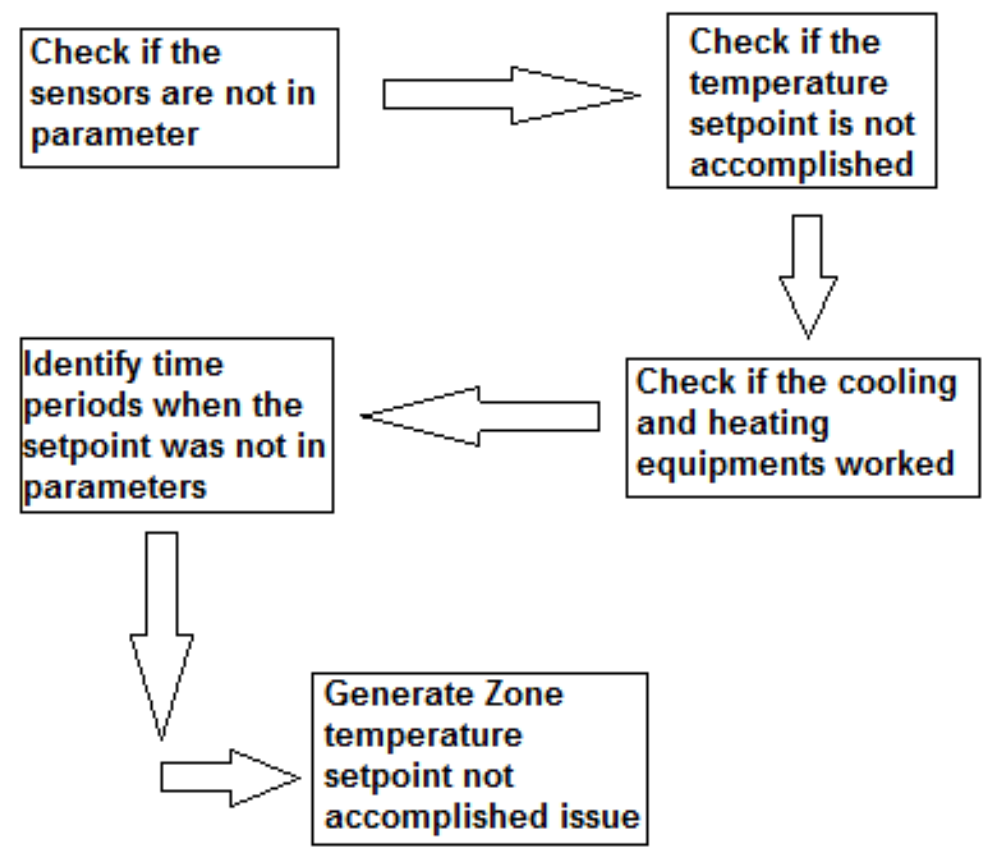

Fig. 3 - Analytic rule process diagram 
The analytic rule was defined in SkySpark analytic software platform. In the first part of the analytical rule, a function is used to check if the temperature sensor and setpoint values are in the parameters and then the rules check if the cooling and heating equipment worked normally. After that, the rules verify the time period when the temperature setpoint was not reached and generate the issues.

\subsubsection{The heating inefficiency analytic rule}

The second defined analytic rule is zone heating inefficiency. This rule checks whether the setpoint temperature is reached when there is a heating demand, when the transitory regime is considered to be finished. If the zone measured temperature is lower than setpoint and the heating actuators has been switched on, it will cause a functional error.

The equation underlying this rule is the following:

where:

$$
\begin{aligned}
& \text { Temperature }+ \text { err }<\text { Heating Setpoint } \\
& \text { err }=\frac{\text { Offset }}{2} \sim 1^{\circ} \mathrm{C} ;
\end{aligned}
$$

Heating Setpoint-the heating temperature request by user;

Temperature-the temperature measured by building temperature sensor;

Offset-the accepted deviation from Setpoint;

The diagram of the heating inefficiency analytic rule process is presented in Fig.4.

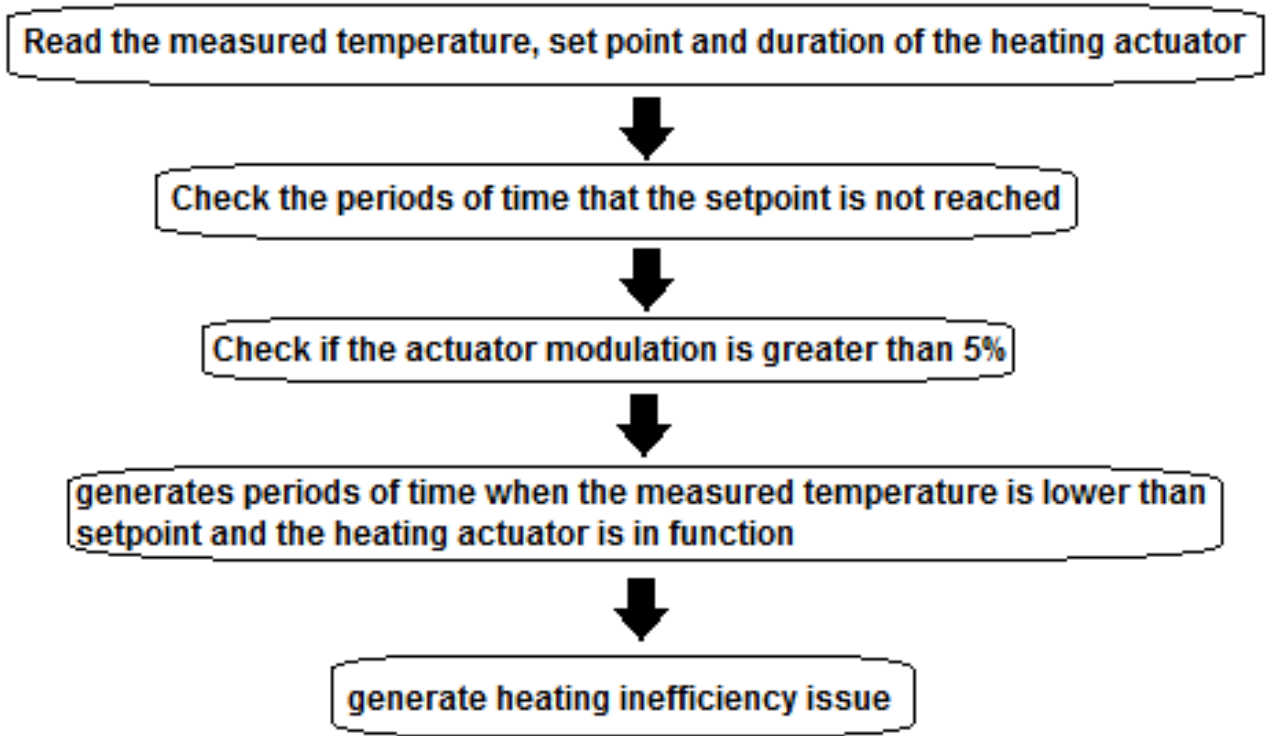

Fig. 4 - Heating inefficiency analytic rule process diagram

In the first part of the analytical rule a function is used to identify the time periods when the measured temperature is lower than setpoint, after that the temperature and setpoint values from cloud database are read and in the end it checks the time periods when the setpoint has not been reached and the issues are generated.

\subsubsection{The cooling inefficiency analytic rule}

The third analytic rule for occupants comfort is Cooling inefficiency. This rule checks if the temperature setpoint has been reached when the cooling request is present, in stationary regime. If the zone temperature measured is higher than the setpoint and the cooling actuator has been turned on, it will cause an issue. 
The equation underlying this rule is the following:

where:

Temperature+err $>$ Cooling Setpoint

$$
\text { err }=\frac{\text { Offset }}{2} \sim 1^{\circ} \mathrm{C} \text {; }
$$

CoolingSetpoint-the cooling temperature request by user;

Temperature-the temperature measured by building temperature sensor;

Offset-the accepted deviation from Setpoint;

The diagram of the cooling inefficiency analytic rule process is presented in Fig.5.

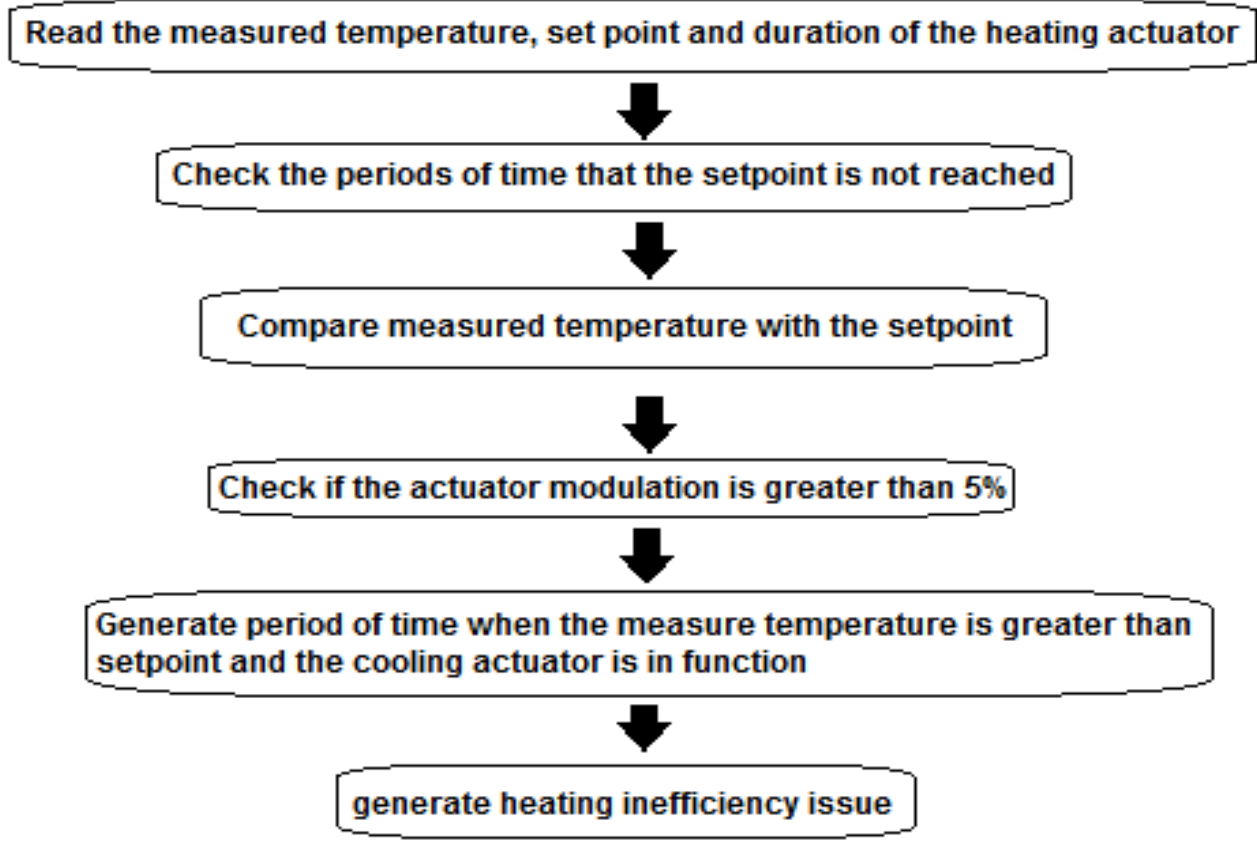

Fig. 5 - Cooling inefficiency analytic rule process diagram

In the first part of the analytical rule a function is used to identify the time periods when the measured temperature is greater than setpoint, after that the temperature and setpoint values from cloud database are read and in the end it checks the time periods when the setpoint has not been reached and the faults are generated.

\subsection{Defined analytic rules related to equipment energy efficiency}

Since few years ago, the increasing building energetics performance and also the cost reduction regarding energy consumption have become important points in managing a building. Having the possibility to collect and manage data from any source, the integration of the equipment energy meters into the cloud software analytics platform will have a very important role in analyzing and/ or improving energy performance of buildings and lowering consumption.

Regarding those mentioned above, an analytic rule which identify if the average energy consumption for a specific day(weekday or weekend) is greater than the last week's, month's or year's average energy consumption (weekday or weekend) by the deviation specified by the building energy manager, was defined.

The equation underlying this rule is the following:

$$
\left(\text { val-avg) }>\frac{\text { avg*dev }^{*}}{100}\right.
$$


where:

val-specified day energy meter read;

avg-daily average of energy consumption for last wheek, month or year;

dev-the predefined and parameterized deviation value

The process model of the abnormal energy consumption analytic rule process is presented in Fig.6.

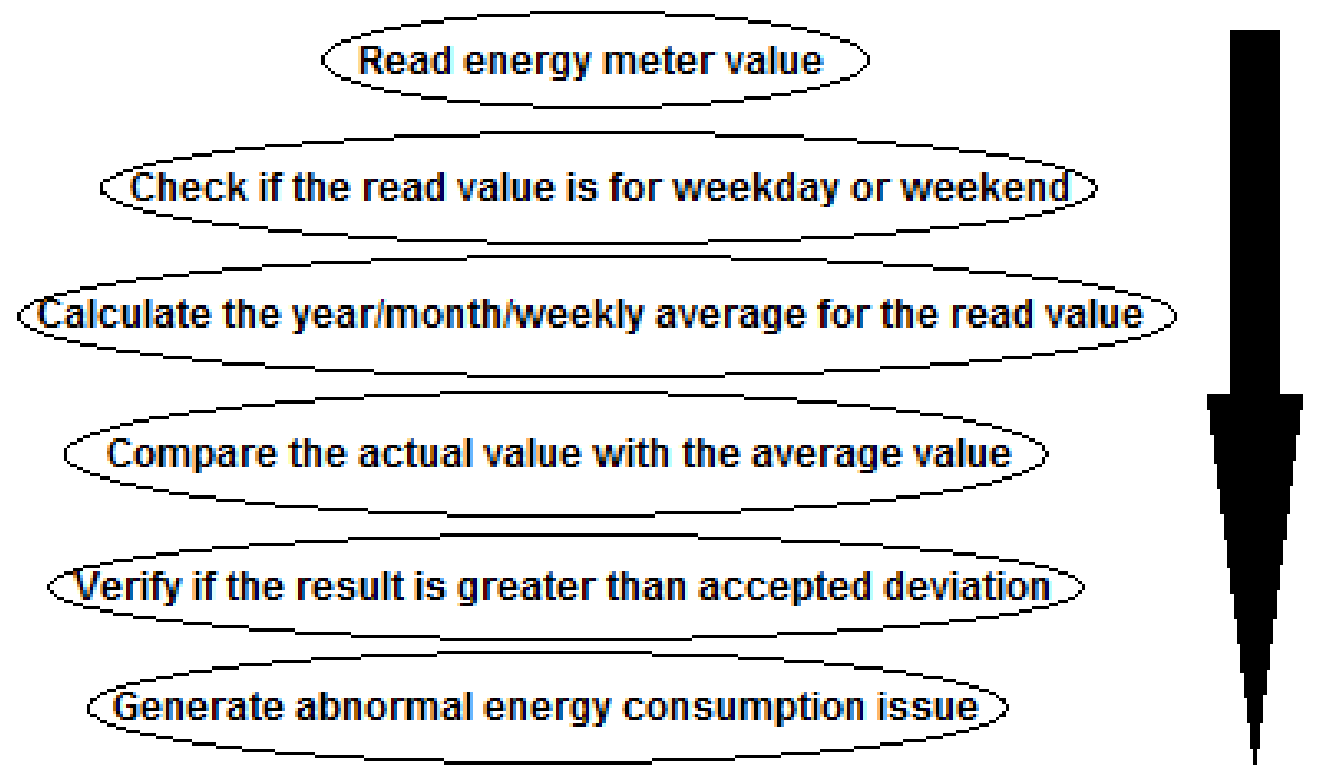

Fig. 6 - Abnormal energy consumption analytic rule process diagram

The logic of the rule is to check if the calculated energy consumption of the day is for a weekday or a weekend and to compare the weekday value with the last month's weekday average or to compare the weekend value with the last month's weekend average. After that, the rule identify if the difference is more than predefined accepted deviation.

The possible causes of the issues can be an inappropriate reading by the energy meter, equipment improper operation or the monitored system is in manual mode.

\section{Case study: applying analytic rules to an on function building}

To check the functionality of the defined software analytic rules related thermal occupants comfort and energy consumption, a series of data is used, taken from an on function building, for one week, having inputs for thermal comfort rule the measured temperature and the setpoint and for energy consumption rules the counted energy consumption. The outputs are represented by the analogical heating and cooling actuators values, to which is added a deviation and the perturbation of external factors.

Applying analytic rules is intended to identify periods when the setpoint temperature has not been reached and the time periods when the energy consumption was greater than the average energy consumption for last week.

\subsection{The result of applying occupants thermal comfort rules}

For the input current temperature and setpoint data taken into the Cloud software platform is presented in table Fig.7. Data was considered for one week. 


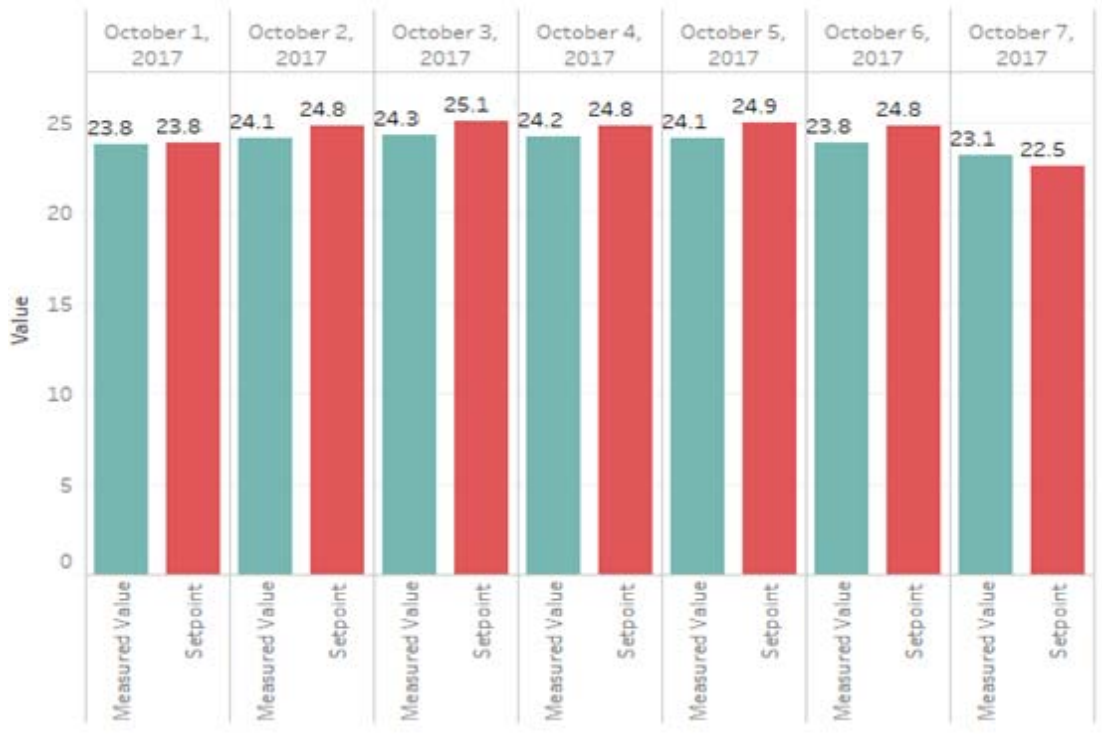

Fig.7 - Indoor temperature parameter data

The resulted issues for a specific day when applying the software analytic rule for heating inefficiency is presented in Fig.8.

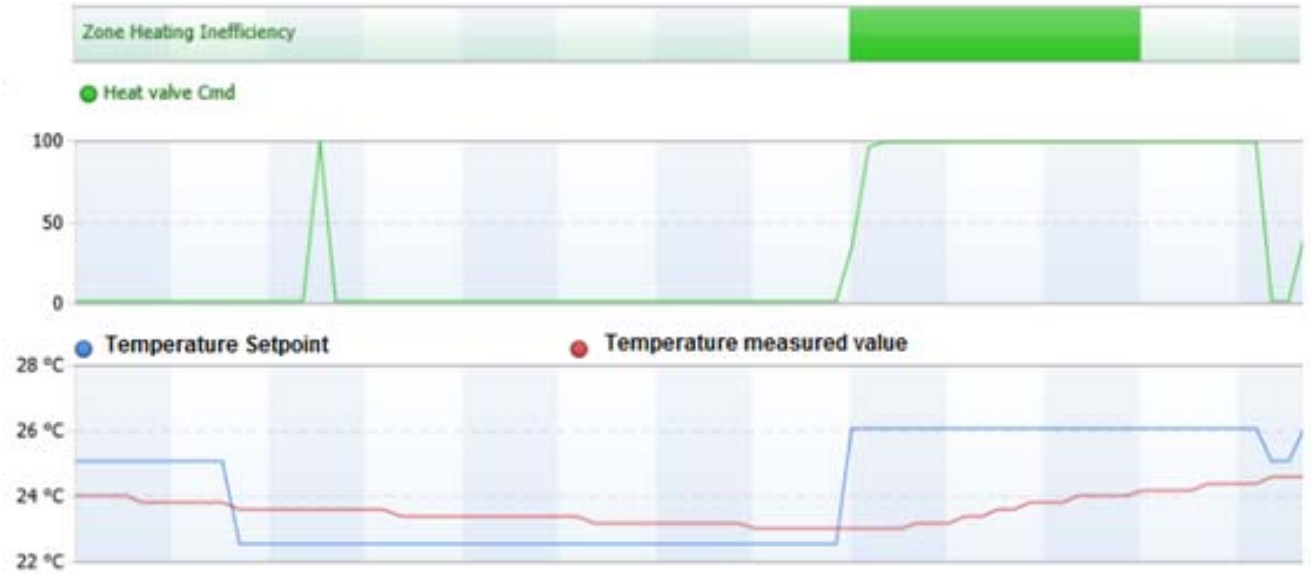

Fig.8 - Heating inefficiency rule results

It can be observe that the rule detects the time periods when the temperature setpoint has not been reached and the heating valve is opened, checked only when the zone state is occupied.

The resulted issues for a specific day when applying the software analytic rule cooling inefficiency is presented in Fig.9.

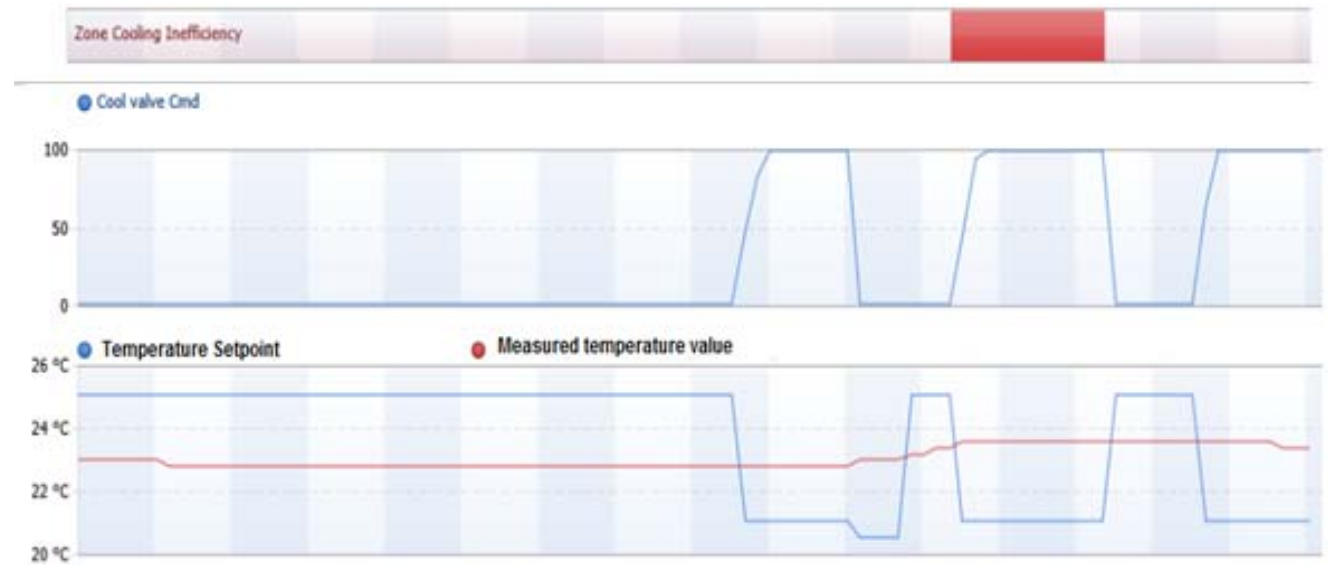

Fig.9 - Cooling inefficiency rule results 
It can be observe that the rule detects the time periods when the temperature setpoint has not been reached and the cooling valve is opened, checked only the zone state is occupied.

The above rules have been applied simultaneously for the data managed and described in Fig.7 Indoor temperature parameter data. The result of the analytical rules can be displayed in a lot of forms and one of the most intuitive of this is the Fig. 10 - Table form results of comfort analytic rules. In this way, the duration of occurrence of anomalies in operation, the number of occurrences of error and the exact time of problem detection are intuitively presented. Also are displayed the affected equipment for each rule and the type of issue (Comfort in this case, but can be Operation or Energy). This graphical presentation of errors allows quick identification of problems and their isolation and immediate correction.

\begin{tabular}{|c|c|c|}
\hline \multirow{2}{*}{$\begin{array}{l}\text { Heating } \\
\text { Inefficiency }\end{array}$} & 3000 ulitin & Heating actuator \\
\hline & 10530u20min & Heating actuator \\
\hline \multirow{4}{*}{$\begin{array}{l}\text { Cooling } \\
\text { Inefficiency }\end{array}$} & $137 \%$ u 37 in & Cooling actuator \\
\hline & 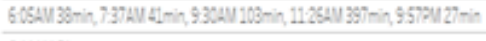 & Cooling actuat \\
\hline & 6114u5:min & Cooling actuat \\
\hline & 7.174U15-in & Cooling actuat \\
\hline
\end{tabular}

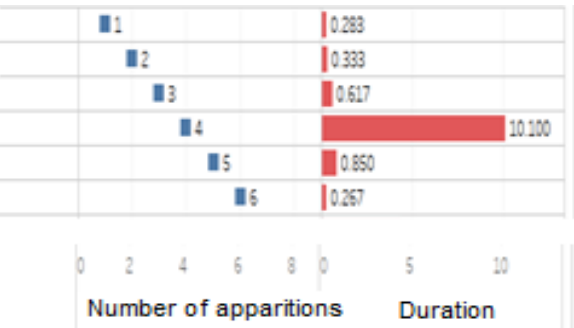

Fig.10 - Table for results of comfort analytic rules

\subsection{The result of applying energy consumption rules}

For the input electric energy consumption data taken from building energy main meter into the Cloud software platform are presented in Fig. 11 - Electrical energy consumption data. Data was considered for one week and the energy measurement units is $\mathrm{kWh}$.

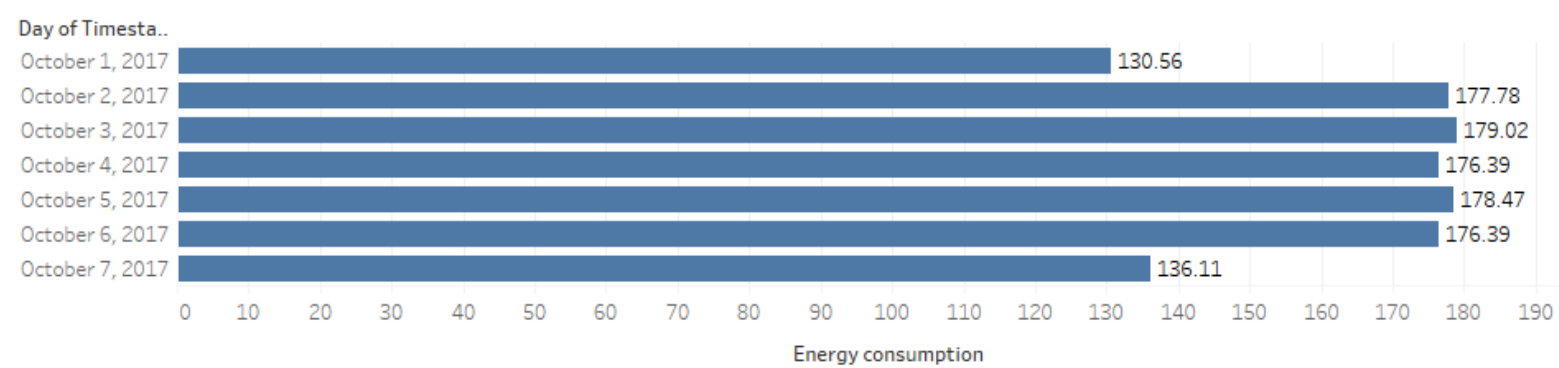

Fig.11 - Electrical energy consumption data

The resulted issues for a specific day, for software analytic rules abnormal energy consumption is presented in Fig. 12.

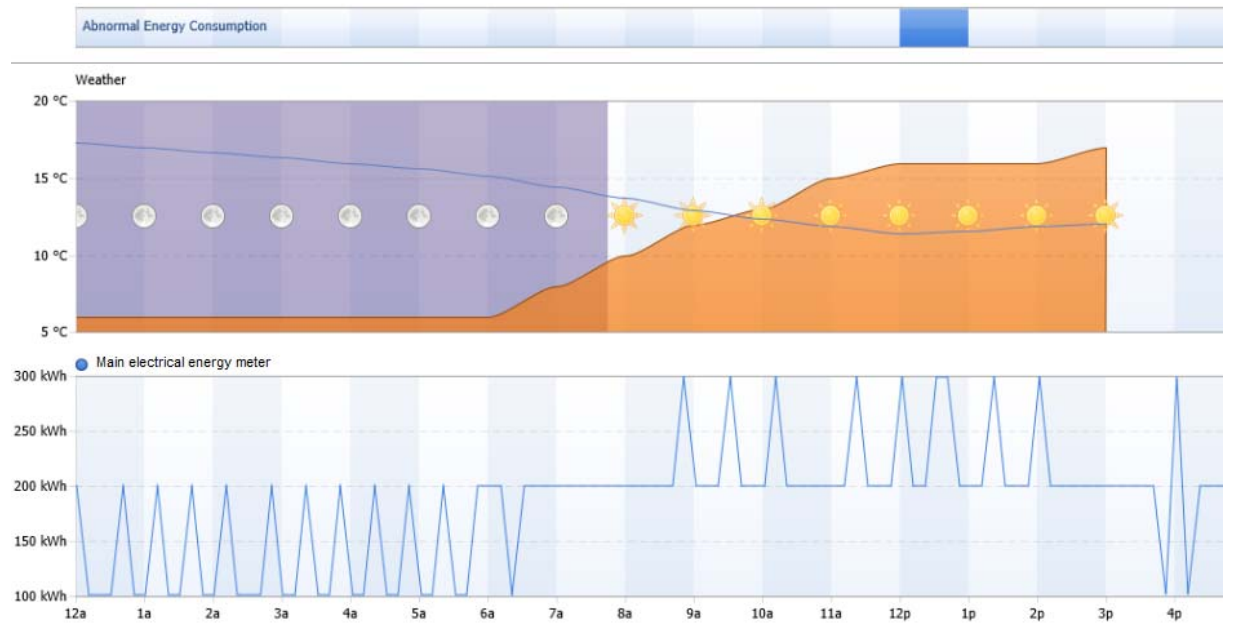

Fig.12- Abnormal energy consumption rule results 
The rule identifies if the average energy consumption of a specific day is greater than the last week average energy consumption by the deviation specified, in this case deviation was defined at $30 \mathrm{kWh}$.

Energy consumption analytic rules help the building managers to have at every time and from everywhere an overview regarding a building energetic performance. The analytic rules warn if very high energetic consumption occurs or if there are inadequate energy meters readings. Also, using energy consumption analytic rules, the equipment which have an inappropriate action in terms of energetic performances can be identified.

\section{Conclusions}

The current state of research and some of the notable achievements in the field of Internet of Things and Cloud Computing applied on building industry are presented in this paper.

Chapter 2 presents the generalized methodology by which data from local controllers for automation systems is transferred to the analysis platforms. A major benefit of data analysis platforms is that once the data is taken into the cloud, it gets released from the system that have collected it. Thus, the data can then be analyzed in several ways using any of the tools that the analyst prefers or thinks most appropriate.

In this paper it was also presented how the software analytics platforms help and increases building comfort and energetic performances. In this purpose, a series of analytic rules regarding the occupants of the building thermal comfort and abnormal energy consumption were developed. These rules have been applied to a real functional building and the results were summary presented and discussed.

The next step in Cloud Computing applied in building industry is to make possible the bidirectional communication between the software analytics platform and the building equipment. Only the data analysis is possible at the moment. The software analytics platform doesn't have the possibility to command the building equipment, but in the nearest future, along with the new research in the Internet of Thing and Cloud computing fields, this will probably be possible.

\section{References}

[1]. Kevin, A. (2009, June). That 'Internet of Things' Thing. Retrieved October 10, 2017, from http://www.rfidjournal.com/articles/view?4986

[2]. Sethi, P. \& Sarangi, S. (2017, January). Internet of Things: Architectures, Protocols and Applications. Journal of Electrical and Computer Engineering. Volume 2017 (2017), 1-25. Retrieved October 09, 2017, from Hindawi database on the World Wide Web: https://doi.org. DOI: 10.1155/2017/9324035

[3]. Iserman, R.(2005). Model-based fault-detection and diagnosis - status and applications. Annual Reviews in Control, 29(1), 71-85. DOI: 10.1016/j.arcontrol.2004.12.002

[4]. Petze, J. (2017, April). Analytics Software for a World of Smart Devices. Retrieved October 07, 2017, from https://skyfoundry.com/file/8/SkySpark-Overview-Brochure.pdf

[5]. Harbor Research (2016, August). The Future of Smart Systems and IoT Analytics. Retrieved October 05, 2017 from https://skyfoundry.com/file/218/Harbor-Research---The-Future-of-IoT-Analytics.pdf

[6]. Project Haystack Standard (2013). Project Haystack Open Source Data Modeling Standard. v2013-10-1. Glen Allen

[7]. Guarino, N. (1997). Understanding, building and using ontologies. International Journal of Human-Computer Studies, 46(2-3), 293-310. DOI: 10.1006/ijhc.1996.0091

[8]. Charpenay, V. (2015, November). Haystack tagging ontology. Retrieved October 02, 2017 from http://vcharpenay.github.io/hto/

[9]. Wurfel, L., Blokker, S. \& Fry,J. (2014). Applying data science to property. Retrieved October 11, 2017 from http://docplayer.net/43491736-Applying-data-science-to-property.html 